\title{
AUTOMATING THE CLASSIFICATION OF STELLAR SPECTRA
}

\author{
CORYN A.L. JONES AND T. VON HIPPEL \\ Institute of Astronomy \\ Madingley Road, Cambridge, CB3 OHA, U.K.
}

We are automating the multi-parameter classification of stellar spectra within the MK system. The increased rate at which spectra are gathered by increasingly larger telescopes, fibre systems and efficient CCD detectors necessitates the need for automation of stellar classification. As well as greatly speeding up this process, an automated scheme would give reproducible results, would arguably be more objective, and would give quick and useful reporting of classification uncertainties.

Our work to date has concentrated on the use of supervised artificial neural networks ${ }^{1}$. Before it can be used to classify spectra, this type of network needs to learn to associate the appearance of a visual spectrum with various spectral parameters. This training process is best performed with a large and comprehensive set of pre-classified training spectra, so as to maximise classification reliability. For this purpose we are using spectra obtained as part of the Michigan Blue Survey ${ }^{2}$, along with the 2-dimensional MK classifications obtained by Houk ${ }^{3}$. To date, we have scanned $\approx 50$ of the original Michigan objective prism plates using the Automatic Plate Measuring facility at Cambridge. Using software we have developed we can extract and reduce the spectra on these plates into a form suitable for input to the neural network. We are currently working on reproducing Houk's luminosity and temperature classifications. Future developments include extension to fainter magnitudes and metalicity classification.

As part of this ongoing project we intend to investigate the potential of unsupervised neural networks, an algorithm in which the network finds and distinguishes its own patterns without training from pre-classified stars. This technique may be able to identify previously unrecognised types of stars or highlight previously undetected correlations in stellar spectra.

\footnotetext{
${ }^{1}$ von Hippel, T., et al. 1994, MNRAS, 269, 97

${ }^{2}$ Houk, N., 1983, in Garrison R.F., ed., The MK Process and Stellar Classification. David Dunlop Observatory, Toronto, p. 85

${ }^{3}$ Houk N., Smith-Moore M., 1988, University of Michigan Catalogue of TwoDimensional Spectral Types for the HD Stars, Vol. 4
} 